\title{
The characteristics on Wang Zhen Specific Training load of a world young record Holder men's 20km Race Walker
}

\author{
HOULIN LI", a , CONG GAO ${ }^{2, \text { b }}$, QINGHUA GAO ${ }^{1, c}$ and YANGYANG,1,c \\ ${ }^{1}$ Capital University of physical education and sports, china \\ ${ }^{2} x i$ 'an physical education university, china \\ alihoulin@126.com, ${ }^{b} 43450102 @ q q . c o m l, 9 h 168043 @$ sina.com
}

Keywords: Training load; Race walker; Specific Training; Wang Zhen

Abstract. To study the Specific Training load of Wang Zhen, Training content was analysis during Preparation for the Tryout of World championship in Athletics. Conclusions: Wang Zhen training is a single-cycle training program, one day Training two sessions, and half-day weekly rest on Sunday. The mainly Training content of Race walking event is technical training, special strength training, functional training and special techniques training. The intensity of training load was divided into four level: General aerobic speed, Specific aerobic speed, Aerobic and anaerobic speed and Anaerobic threshold speed The mainly task of training is developing of training Race basis aerobic capacity and special techniques skills training General Preparation stage. Maintain the aerobic capacity, increasing the amount of training the anaerobic threshold intensity in Specific preparation stage. Reducing of the amount of aerobic speed training load is mainly means to promote recovery the amount of anaerobic threshold speed training increased slightly in Pre-Competition Preparation stage.

\section{Introduction}

Race walking is contested at all major athletics championships. It is an event requires great endurance and ability. The exaggerated gait used is a function of the rules that govern the event (Cairns, Burdette, Pisciotta, \& Simon, 1986). The specific endurance is the key factor that race walker success in competition, once the race walker is perfect in technical. Most of article is study on the race walking technique. There is little training load research on the race walkers, and in particular elite competitors. For it is difficult to acquire the training load information about an elite race walker. New research on these athletes in competition is therefore crucial to understanding the endurance demands of the event. Wang Zhen is height 1.8meter weight 62 kilogram and birth day is 24 august 1991.Wang Zhen broke the young men's $10 \mathrm{~km}$ race walk world record with 37:44 at the In 2011 IAAF Race walking challenge in Bei Jing on 18 SEP 2010.He won the first place of men 20km Race Walking in Tryout of IAAF World Race Walking Championships.It is also important to write down the training load and training content during preparation for the tryout of World championship .

\section{Methods}

Table 1 Training time

\begin{tabular}{lllllc}
\hline Start Training & $\begin{array}{l}\text { The Tryout of World } \\
\text { championship }\end{array}$ & Total km. & $\begin{array}{l}\text { Training } \\
\text { sessions }\end{array}$ & Weeks & Days \\
\hline 16 December2010 & 24April 2011 & 1522.32 & 228 & 17 & 133 \\
\hline
\end{tabular}

2010 Wang Zhen was elect to china race walking team which coach come from Italian whose athletes acquired Olympic champions. Wang Zhen won the championship on IAAF Race walking challenge at Switzerland Lugano on .march 2011 time 1:20:42.It is 1:28 shorter than his PB 1:22:10. As a researcher on the china national race walking team, we have tracked the training process and record his training content and training load. From Wang Zhen winter training start which was preparation for the World championship competition On December 16, and finish on the competition day .It is benefit for scientific research team to study on the elite race walker. 
Treadmill and Road race walking training Volume record unit as $\mathrm{km}$, Mountaineering training Volume record unit as km. bicycle exercise and fitness training Volume record unit as house

\section{Data analysis}

The Start Training time of Wang Zhen preparation for the Tryout of 13th IAAF World Championships in Athletics training period is 16 December 2010, The end Training time is 24April 2011.Which is The Tryout of 13th IAAF World Championships. Total of 133 days 17 weeks (Table 1).Training time is 108 days and $81.20 \%$ of total days. According to the coach training plan It is mainly to training Injury and Trip time is respective 3 days in this training period, It is only $2.2 \%$, which is less than contest days (Sheet 2). There are 228 Training sessions in 133 day, which is Average 1.7 Training sessions one day, when trip days, Injury days and contest days are get rid . It is 1.9 Training sessions average day, Wang Zhen have two Training sessions and rest time in afternoon of Sunday. Training volume is 1522.32 kilometer. Therefore this Preparation training stage is systematic training.

Table 2 Training information (day)

\begin{tabular}{lllllll}
\hline & Training & Rest & Contest & Injury & Trip & Total \\
\hline Day2 & 108 & 12 & 7 & 3 & 3 & 133 \\
percent $(\%)$ & 81.20 & 9.02 & 5.26 & 2.26 & 2.26 & 100.00 \\
\hline
\end{tabular}

The training stage of Wang Zhen: There are four training stage in which Wang Zhen preparation training for the tryout of World championship period. The first stage of training time is from 16 November 2010 to 26 November 2010, 11 days was training in Nanjing. The mainly training scheduled task is Body recovery. Was calling recovery Phase. Mainly aim is constructive and recovery training, including force training, flexibility training, coordination training content

The second stage of training time is from 27 November 2010 to 6 February 2011, 43 days was training in Nanjing. The mainly training scheduled task is .to increased aerobic durance was calling General Preparation stage, Mainly aerobic endurance training, strength training, flexibility training, posture and coordination training content. The third phase of training time is from 7 February 2011 to 4 March 2011, 26 days was training in Italia Saluzzo - San Lorenzo. The mainly training scheduled task is to increase aerobic durance and Special endurance was calling Specific Preparation stage.

The fourth stage of training time is from 4 April 2011 to 24 April 2011, 20 days was training in Nanjing. The mainly training scheduled task is to increase Special endurance and Cultivate competition state was calling Pre-Competition Preparation stage. The results show that it is Single cycle training in the course of preparation tryout of World championship. Wang Zhen training is a single-cycle training program, one day Training sessions, and half-day week rest on Sunday.

Table 3 Training content Summary

\begin{tabular}{lll}
\hline Type & Means & Statistical unit \\
\hline Road race walking training & variety of speed walking & Kilometer $(\mathrm{km})$ \\
Mountain climbing & Walking & Kilometer $(\mathrm{km})$ \\
Treadmill & Stretch, technology & Kilometer $(\mathrm{km})$ \\
Exercise bikes & Bike & Kilometer $(\mathrm{km})$ \\
Body function training & Core strength training, coordination & house $(\mathrm{H})$ \\
\hline
\end{tabular}

Wang Zhen Training content: Training content of Wang Zhen include Road race walking training, climbing training, treadmill training, core strength training and coordination exercises and flexibility exercises and biking training. The coach classified the core strength training, coordination exercises and flexibility exercises as the body function training statistical. Statistical unit house.. Road race walking training, and climbing training, basic statistical unit is kilometer. Road race walking training is race walking event Specific basically training means. climbing training is race walking event Specific strength training means, especially it is benefited to the push amplitude ; biking training is vital to the race walker support strength. Thus climbing training and biking training is the race walking event 
Specific strength means. Treadmill training is good for race walking to improverace walking techniques.

Table 4 Wang Zhen Training volume average Weeks statistics $(\mathrm{km})$

\begin{tabular}{llll}
\hline Stage & Road race walking & Mountain climbing & Treadmill Training \\
\hline General Preparation stage & 91.88 & 3.75 & 4.75 \\
Specific Preparation stage & 106.94 & 5 & 3.38 \\
Pre-Competition stage & 82.67 & 3.33 & 0 \\
\hline
\end{tabular}

In a word briefly race walking event training content was divided into techniquestraining, Specific strength means, body function training and race walking training. The volume of Wang Zhen road race walking training load average week .:The volume of road race walking training is 1075 kilometer. $93.3 \%$ of total training (table 4). It is the basically capacity for race walking to promote the level of sports ability. maximum of Wang Zhen road race walking training volume is $106.94 \mathrm{~km}$ weekly in special preparation stage. The general preparation stage is $15.06 \mathrm{~km}$ less than special preparation stage weekly .equivalent to $85.9 \%$ of special preparation stage; which is $91.88 \mathrm{~km}$ weekly, minimum of The Pre-Competition Preparation stage is $82.67 \mathrm{~km}$ weekly. The Pre-Competition Preparation stage is less than $24.27 \mathrm{~km}$ weekly, equivalent to $77.4 \%$ special preparation stage. As we can showed that the volume of road race training is maximum, the general preparation stage is less, and The Pre-Competition Preparation stage is minimum.

Mountain climbing is a basically Specific Strength Training means (Table 4), which carry out the whole training process. The maximum volume of Wang Zhen Mountain climbing is $5 \mathrm{~km}$ weekly in special preparation stage. The general preparation stage is $3.75 \mathrm{~km}$.It is less $1.25 \mathrm{~km}$ weekly than special preparation stage. Equivalent to 75 percent of special preparation stage; The Pre-Competition Preparation stage is minimum of $3.33 \mathrm{~km}$ weekly. The Pre-Competition Preparation stage is less than $1.67 \mathrm{~km}$ weekly, equivalent to $67 \%$ special preparation stage. The results show that the volume of Mountain climbing is stability in three preparation stage.

Table 5 The race walking speed and training intensity load

\begin{tabular}{lll}
\hline & Speed( per kilometer) & Training Intensity \\
\hline General & $>5$ minutes 20 second & $<75 \%$ \\
aerobic & 5 minutes 20 second - 5 minutes 10 second & $75-77 \%$ \\
speed & 5 minutes 10 - 5 minutes & $78-80 \%$ \\
\hline Specific & 5 minutes - 4 minutes 50 second & $80-83 \%$ \\
aerobic & 4 minutes 50 second - 4 minutes 40 second & $83-86 \%$ \\
speed & 4 minutes 40 second - 4 minutes 30 second & $86-90 \%$ \\
\hline Aerobic & 4 minutes 30 second - 4 minutes 20 second & $90-92 \%$ \\
and & 4 minutes 20 second - 4 minutes 10 second & $92-96 \%$ \\
Anaerobic & 4 minutes 10 second - 4 minutes & $96-100 \%$ \\
speed & Anaerobic & \\
Threshold & $<4$ minutes & $>100 \%$ \\
speed &
\end{tabular}

Treadmill Training is one Race walking Special technique training means (Table 4), which is used in General Preparation stage and Specific Preparation stage. It is a mainly training means to improved race walking technique. The volume of Wang Zhen Treadmill Training is $4.75 \mathrm{~km}$ weekly General Preparation stage. It is take longer time to improve Wang Zhen sports techniques. The volume of Treadmill Training is $3.38 \mathrm{~km}$ weekly in special preparation stage.There are no Treadmill Training in Pre-Competition stage. Variety of Race walking training on the track and field was used to improved the race walking in order to Adapt to the competition. The intensity of Wang Zhen training load: Wang Zhen $20 \mathrm{~km}$ race walking Performance time is 1:20: 42 in 2010. The aimed time of Wang Zhen20 km race walking is 1:20:20, the average speed is 4 minutes per kilometer. It is Reasonable performance 
goal for Wang Zhen. He won the first place in the Tryout of 13th IAAF World Championships in Athletics. Time is 1:18:30. 4 minutes per kilometer speed is 100\% intensity load in the coach statistical

(table5). And the race walking training speed was divided into ten speed level by interval 10 second per kilometer. In other words the speed is from 4 minutes per kilometer to 5 minutes 20 second per kilometer (Table 5).

Table 6 Wang Zhen Training volume percent in different stage (\%)

\begin{tabular}{l|llll}
\hline Stage & $\begin{array}{l}\text { Aerobic } \\
\text { speed }\end{array}$ & $\begin{array}{l}\text { Aerobic and } \\
\text { anaerobic } \\
\text { speed }\end{array}$ & $\begin{array}{l}\text { Anaerobic } \\
\text { threshold } \\
\text { speed }\end{array}$ & $\begin{array}{l}\text { Treadmill and } \\
\text { mountain } \\
\text { climbing }\end{array}$ \\
\hline General Preparation stage & 87.67 & 3.86 & 0 & 8.47 \\
Specific Preparation stage & 73.55 & 13.44 & 5.75 & 7.26 \\
Pre-Competition Preparation stage & 72.66 & 13.31 & 10.43 & 3.60 \\
\hline
\end{tabular}

The speed is fast than 4 minutes per kilometer named anaerobic threshold speed for $20 \mathrm{~km}$ race walking. The speed is named aerobic and anaerobic speed from 4 minutes per kilometer to 4 minutes30second per kilometer and the intensity of training is $100 \%$ to $90 \%$ of the aiming speed.The speed is named Specific aerobic speed from 4minutes30second per kilometer to 5minutes per kilometer and the intensity of training is $90 \%$ to $80 \%$ of the aiming speed.The speed is named General aerobic speed from 5minutes per kilometer to 5minutes30second per kilometer and the intensity of training is $80 \%$ to $75 \%$ of the aiming speed. The coach said that the speed is slowly than 5 minutes 20 second is invalidation training content.

Table7 Wang Zhen Training volume percent

\begin{tabular}{cccc}
\hline & $\begin{array}{c}\text { Intensity of } \\
\text { Training load }\end{array}$ & $\begin{array}{c}\text { The volume of } \\
\text { training }\end{array}$ & percent $\%$ \\
\hline General aerobic speed & $<75 \%$ & 422 & \\
& $75-77 \%$ & 210 & 45.25 \\
& $78-80 \%$ & 274.5 & \\
Specific aerobic speed & $80-83 \%$ & 269 & 33.84 \\
& $83-86 \%$ & 273.5 & \\
Aerobic and anaerobic & $96-90 \%$ & 135.5 & \multirow{2}{*}{-5.58} \\
speed & $90-92 \%$ & 96 & \\
Anaerobic threshold & $96-100 \%$ & 48 & 4.09 \\
speed & $>100 \%$ & 82 & \\
\hline
\end{tabular}

The results show that there are five intensity of Training load Zones in the course of Wang Zhen Preparation for the Tryout of 13th IAAF World Championships in Athletics

Wang Zhen training load in General Preparation stage: The volume of Aerobic speed training is $87.67 \%$ of total training in the course of General Preparation stage(Table6). It is more than the training plan for Wang Zhen was not suitable . Most of speed is 6 minute per kilometer. There are $422 \mathrm{~km}$ which speed is lower than 5 minutes 20 second per kilometer in the course of Preparation stage (Table7), equivalent to the volume of 5 minutes 20 second - 5 minutes per kilometer.

The volume of Aerobic and anaerobic speed training is $3.86 \%$ of total training in General Preparation stage(Table6), there are no volume of Anaerobic threshold speed training. Wang Zhen took long time to improve race walking techniques and increased Specific strength. Volume of Treadmill and mountain climbing training is double of Aerobic and anaerobic which is $8.47 \%$.

Wang Zhen training load in Specific Preparation stage: The volume of Aerobic speed training is decreased from $80.5 \mathrm{~km}$ to $78.6 \mathrm{~km}$. percent from $87.67 \%$ to $73.55 \%$ of total training in the course of Specific Preparation stage (Table6).It is Reduce $2 \mathrm{~km}$ than and General Preparation stage. Wang Zhen won first place in AAF race walking challenge Swiss time is 1 hous 18 minutes 37 second .20 March 
2011 during the Specific Preparation stage. The volume of anaerobic threshold speed training is increased, which is $5.75 \%$ Specific Preparation stage.

Wang Zhen training load in Pre-Competition Preparation stage: The mainly task is to adjust physical condition in Pre-Competition Preparation stage: The volume of Aerobic and anaerobic speed road race walking training is $80 \mathrm{~km}$ weekly in Pre-Competition Preparation stage. The volume of aerobic speed training is only $60 \mathrm{~km}$, reduced by $20 \mathrm{~km}$ weekly; it is only $77 \%$ of the specific preparation stage. The volume of Aerobic and anaerobic speed race walking training total training volume decline of $85.8 \%$ weekly. The results show that the volume of race walking training reduced by $20 \mathrm{~km}$ weekly. The aerobic and anaerobic speed race walking training load is reduced is the main training content in Pre-Competition Preparation stage. The volume of Aerobic and anaerobic speed race walking training is accounted for $13.31 \%$ of total training in Pre-Competition Preparation stage .It is equal to the special preparation stage. The volume of training in preparation by the $14.3 \mathrm{~km}$ in Specific Preparation stage weekly down to $11 \mathrm{~km}$ weekly in Pre-Competition Preparation stage, The volume of race walking training reduced by $3.3 \mathrm{~km}$ weekly. The volume of Anaerobic threshold speed race walking training increased from $6.1 \mathrm{~km}$ weekly in Specific Preparation stage to $7.6 \mathrm{~km}$ weekly in Pre-Competition Preparation stage. Although there are a lot of race walking technical training to improve the special race walking, The volume of the anaerobic threshold speed training, increased by $1.5 \mathrm{~km}$ average week.

\section{Conclusions}

Wang Zhen training is a single-cycle training program, one day two Training sessions, and half-day weekly rest on Sunday. The mainly Training content of Race walking event is technical training, special strength training, functional training and special training. The intensity of training load was divided into four: General aerobic speed, specific aerobic speed, Aerobic and anaerobic speed and anaerobic threshold speed. The mainly task of training is to develop training Race basis aerobic capacity and special technical skills training General Preparation stage. Maintain the aerobic capacity, increasing the amount of training the anaerobic threshold intensity in Specific preparation stage .Reducing the amount of aerobic speed training load is mainly means to promote recovery the amount of anaerobic threshold speed training increased slightly in Pre-Competition Preparation stage.

\section{References}

[1] Bland, J.M., Altman, D.G.. Statistical methods for assessing agreement between two methods of clinical measurement. Lancet, i(8476), 307_310

[2] Arcelli, E. (1996). Marathon and 50km race walk: physiology, diet and training. New Studies in Athletics, 11(4), 51_58

[3] Hopkins, J. Improving performance in the 50 kilometreswalk. New Studies in Athletics, 5(3), 45_48

[4]http://www.iaaf.org/athletes/pr-of-china/zhen-wang-240586 